\title{
Search Engine Optimization as a Passive Technique to Promote Business
}

\author{
Shashi Prakash Agarwal \\ Mpstme, \\ Svkm's Nmims, \\ Shirpur, Mh, India
}

\author{
Upendra Verma \\ Mpstme, \\ Svkm's Nmims, \\ Shirpur, Mh, India
}

\begin{abstract}
A key major expenditure for any business in existence is advertisement and presence in widespread media. This paper describes possibilities of empowering business with help of algorithms that can benefit it with a higher search engine response position. Internet has a much wider reach than all of traditional medium of communication combined. This reach can be harnessed to improve growth rate of a company.
\end{abstract}

\section{General Terms}

SEO: Search Engine Optimization; SME: Small and medium sized enterprises; SERP: Search Engine Response Position

\section{Keywords}

Search engine optimization, search engine response position, business growth hack, internet marketing techniques

\section{INTRODUCTION}

SMEs are growing at an exponential rate around the globe and seek a cost-effective method of marketing their products / services. Considering the recent layoffs in tech startups led to the development of this research paper which focuses on effective utility of search engine optimization in the long run.

\section{INTRODUCTION TO SEARCH ENGINE OPTIMIZATION}

Search Engine Optimization refers to several on and off page techniques that help improve the overall appearance of a targeted web resource in a search engine in context with predetermined keywords or related. There is no definite or exact pattern that leads to a higher search engine response position. Google remains a favorite source of traffic for bloggers and has over 300 algorithmic changes in its query response mechanism throughout a year. This makes it impossible to fix a scalable strategy that may work for every website. The process of getting traffic from free / natural result pages on a search engine encompasses several techniques such as rewriting meta keywords \& description, backlink creation and content marketing.

The most appropriate way to start with Search Engine Optimization is to start with a tool like Google Webmaster's Central / Bing's Webmaster Central. Upon successful verification of the portal, one can get access to list of keywords that gets them featured on search engines. This helps in planning content for higher results. Such tools also show details such as potential keywords, list of sites linking to the web resource. The most appropriate way to start with Search Engine Optimization is to start with a tool like Google Webmaster's Central / Bing's Webmaster Central. Upon successful verification of the portal, the webmaster gets access to list of keywords that gets their portal featured on search engines. This helps in planning content for higher results. Such tools also show specific and in-depth details such as potential keywords, list of sites linking to you and various other aspects that affects the position of website for any query.

Instead of taking search engines as just a different element of web, considering it as a normal 'user' will help build a higher impact. There is no point in optimizing websites for search engines and not users. The real goal of any marketing campaign is to generate leads that may turn into real conversions. Prime purpose of this technique is to reach actual users and not just a looped collection of search engine crawling bots.

Following factors play a key role in SERP:

- Title of Webpage

- Relevance of meta description \& keywords

- Value of content for user

- Number of authority links to the web site

- Page Load Time

- Social Media Reach

- Structure of URL / Navigation

- XML Sitemap

- Alt attribute for images

- Headings

- Robots.txt

- Mobile compatibility

\section{BASICS OF SEARCH ENGINE OPTIMIZATION}

\subsection{Title of a web page}

A title tag on a webpage needs to be related to the content of the web page. This helps search engines to reference to the web page when a similar pattern of keywords is used in a query and the web page's title. However, maintaining a unique title for every page on the website is absolute necessary. Furthermore, one cannot just target search engines because a title is also a visible element of a web page. Any user browsing a particular webpage sees its title at the top of the browser. It needs to be necessarily relevant. It is strongly warned to not simply stuff keywords in an unnatural form or the effectiveness on search engines may drop significantly.

\subsection{Meta description and keywords}

Quality and original content is a key factor that gains visitors. However, meta description and keywords convey the necessary meaning to search engines in comparatively less cluttered form. One of the most initial step to SEO is to write meta description and keywords for web pages. This can be 
written with tags like <meta name="keywords" content $=$ " your,set,of,keywords"> and <meta name="description" content $=$ "Precise description of the content of the web page " $>$. If the meta tags are managed well, one can expect a decent response without much of off-page optimization. However, as this is a basic technique - it is widely used and hence very competitive to find the right set of keywords and description.

\subsection{Value of Content}

It is necessary to understand that people would never be interested in plagiarized content. Instead they need access to improved knowledge on a set of keyword which didn't exist previously. Inventing valuable content is an important part of optimization. If the content being marketed doesn't add value to life of customer, they would never be interested in reading it. That is why when a software product is built, online support forum comes to a great rescue as the users will be engaged throughout their session on the website.

Tools such as Copy Scape can be implemented to verify if a particular post is copied from some other website or potential source of content. And if it is not possible to invent new content then it is required to backlink to the source of information as a reference while adding their work on the web page. One needs to track activities of user using tools like Google Analytics and Kissmetrics to find out where the user navigates on his first visit. This will give access to mind set of most of the targeted users.

Suppose, a navigation bar at the top attracts 200 clicks while that at the footer attracts 50 clicks - it is advisable to place higher grade content at the top rather than at the footer.

Certain search engine optimization practices may restrict the portal to a minor extent about location of links in a HTML page but it should never hinder the user experience. One should remember that people will be using their website and not search engines. Hence, user experience should be optimum.

\subsection{Number of authority links to website}

This remains as a key to success as far as Google is concerned. The number of websites linking to the targeted website determines relative position with competitors. However, it should be noted that bulk link harvesting tools should never be used -as they get the website penalized by search engines on account of hyper spamming to obtain higher results. Search Engine Optimization is a medium or long term goal and takes time to execute effectively. It is extremely necessary to maintain that sites linking to target website do not contain spam. Search engines detect black hat techniques by rating growth rate of backlinks.

Suppose a website A had 500 backlinks last Monday and by Thursday, it grows to 10,000 without necessary quality content / article backing the link. This may bring a suspicion amongst search engine crawlers and the target site may end up spoiling its appearance on search engines.

Alternatively, webmasters can approach other webmasters in common niche to back link to published content. Convincing a webmaster to do that may require monetary inputs but a relevant back link adds more value to content than ten back links from websites with poor content. It can be understood this from the illustration.

\begin{tabular}{|l|l|l|l|l|}
\hline $\begin{array}{l}\text { Web } \\
\text { Site }\end{array}$ & Backlinks & $\begin{array}{l}\text { Details about } \\
\text { Backlinks }\end{array}$ & Positions & $\begin{array}{l}\text { Alexa } \\
\text { Rank }\end{array}$ \\
\hline A & 7,500 & Bulk / Spam & 7.1 & $19,00,000$ \\
\hline B & 800 & $\begin{array}{l}\text { Genuine / } \\
\text { Backed by } \\
\text { strong article } \\
\text { base }\end{array}$ & 3.1 & $5,00,000$ \\
\hline C & 24,000 & $\begin{array}{l}\text { Genuine / } \\
\text { Backed by } \\
\text { strong article } \\
\text { base and } \\
\text { higher user } \\
\text { engagement. }\end{array}$ & 2.2 & $\begin{array}{l}\text { Within top } \\
50,000\end{array}$ \\
\hline
\end{tabular}

3.5 Website Load Time

The amount of time a website takes to load in an average internet connection determines the bounce rate of a visitor. For an average user, a load time higher than 3 seconds may lead to loss in interest \& no further usage. An average user expects a web page to load in $2-4$ seconds at the most.

To reduce website load time, reduction is required in context with the number of HTTP requests generated and size of various resources used on the website - it may include images, javascript, CSS files and response time of server side scripts.

The load time of a webpage can be checked using Google's Speed Light.

\subsection{Social Media Reach}

With introduction of social-networking rich content on web pages, it is important to consider social media count as a key factor in response position. A higher share by a public profile on social networking platform such as Facebook may help an organization bring a page to a higher rank on search engines as well. It is important to consider role of Google Plus when dealing with Google - the king of search engines.

\subsection{Structure of URL}

The structure of URL plays a role similar to title of a website. Any content on web is referred with a URL and if this URL contains targeted keywords of relevance, it may attract more people.

Example:

Suppose the following URLs: http://[nameofyoursite.com]/dev/struct/console.html

$\&$ http://[nameofyoursite.com]/dev-console. It is very easy to perceive that the second URL is easier to memorize or use for an average human with a nominal IQ. Similarly, improved visibility is observed when the target URL is highly optimized.

\subsection{XML Sitemap}

Sitemap is an XML file that contains list of URLs to be indexed by a search engine. A software such as XML Site ap generator may be used to index links visible on a website and the generated XML file has to be uploaded to the respective web master central consoles such as Google Web Master Central. Open Source CMSs are pre-built with modules that allow this to happen.

\subsection{Alt attribute for images}

Frankly search engines are blind about images and seek alternate text in place of images. It is very important to use alt 
attribute for images as this may help target more relevant keywords.

Example: <img src="graphics.png" alt="My First Book">

\subsection{Heading}

The headings used on the webpage also contain an influence over the SERP. The frequency of using heading tags may boost or reduce overall appearance. You need to format and draft the curated content in an easy to understand format so as to rank better.

\subsection{Robots.txt}

Robots.txt is a file that contains permission for user agents to access various resources on the website. It is required to allow search engine bots to index web pages in a website and this is possible with Robots.txt. Without an effective robots.txt, the entire process of search engine optimization may turn out to be useless as the crawler may never be able to index the target resources. This may restrict any further interaction of search engine with $a b$ interface. Robots.txt contains list of permissions for search engines / user agents.

\subsection{Mobile Compatibility}

Several people use smartphones and not desktops / laptops on a more regular basis and missing mobile compatibility may badly hamper the customer base. It is important to make a mobile version of website as well - to reach the smartphone users within a limited bandwidth. Moreover, the design should be responsive yet simple without excess of navigation links.

\section{FUTURE SCOPE}

Search Engine Optimization is a tool for the visionary and knowledgeable person to market their products really well. It is necessary to consider real users rather than just search engines.
The day content on a website benefits its users; search engines will start upgrading their position on search engine queries. This shall be achieved with quality content, meaningful content and meta- tags, stream of back links and user experience.

If the mentioned steps are taken into consideration, the way to reach a wider population could be easier at minimal cost.

\section{REFERENCES}

[1] Gupta, Sachin, and Ankit Aggarwal. "Study of Search Engine Optimization." International Journal of Research in Engineering \& Applied Sciences 2.2 (2012): 15291536 .

[2] Godhwani, Poonam Bhagwandas. "A research paper on SEO aid in mobile website optimization." International Journal of Managment, IT and Engineering 3.6 (2013): 282-288.

[3] Soleimanian, Farhad, Marjan Mahmoodi Tabrizi, and Isa Maleki. "Search Engine Optimization based on Effective Factors of Ranking in Web Sites: A.(2014) ".weiveR

[4] Silva, Nuno, and António Aguiar. "Web site optimization for search engines: An empirical study." 2014 9th Iberian Conference on Information Systems and Technologies (CISTI). IEEE, 2014.

[5] Rafiq Elmansy, "Search Engine Optimization", John willey \& Sons Inc., 2013.

[6] Jennifer Grappone and Gradiva Couzin, "Search Engine Optimization: An Hour a Day", Wiley Publishin, Inc.2006.ACKNOWLEDGMENTS 